\title{
Insights into the care coordination needs of people living with rare diseases in Western Australia
}

Alicia Bauskis ( $\square$ alicia.bauskis@health.wa.gov.au )

Western Australia Department of Health

Caron Molster

Western Australia Department of Health

Research

Keywords: rare diseases, care coordination, holistic care

Posted Date: March 20th, 2020

DOI: https://doi.org/10.21203/rs.3.rs-17749/v1

License: (c) (i) This work is licensed under a Creative Commons Attribution 4.0 International License. Read Full License 


\section{Abstract}

\section{Background}

People living with rare diseases face complex medical needs as well as a range of non-medical care needs. However, there is limited research on the experiences of care coordination for those living with a range of rare conditions. The paper addresses a research gap by capturing the views of both parents of children living with RD and adults living with rare diseases, and also explores some factors affecting people living in regional areas.

Three focus groups were conducted with a total of sixteen participants who were adults living with a rare disease (and one carer) and parents of children living with rare diseases in Western Australia. Results were analysed using Braun and Clarke's thematic analysis.

Results

The study revealed that the participants in our study who were living with a rare disease (or their carers) (1) have complex care needs and a need to access multiple systems (2) have mixed experiences of care coordination (3) often go above and beyond to self-coordinate their care; and they (4) recognise opportunities for more systematic care coordination.

\section{Conclusion}

Our study has highlighted the complexity of care needs for many people living with a rare disease and the need for care coordination both within and across medical and non-medical domains. In exploring opportunities to provide more systematic care coordination, consideration should be given to European models of Centres of Expertise for Rare Diseases as well as complementary Resource Centres. Consideration of both models will help ensure patients receive holistic, well-integrated medical and nonmedical care.

\section{Background}

Rare diseases are commonly "life-threatening or chronically debilitating diseases which are of such low prevalence (1 in 2,000 people) that special combined efforts are needed to address them" [1]. This statement is used by the European Commission to guide their understanding of rare diseases and it underpins the Western Australia Rare Diseases Strategic Framework 2015-18 (the WA Framework), the first government plan created for rare diseases in the southern hemisphere [2]. The WA Framework also recognises that many rare diseases cannot be prevented, are complex with multi-system dysfunction, disabling, incurable and have no effective treatment $[3,4]$. While the prevalence of each rare disease is individually low, there are an approximately 6,000 to 8,000 different types of rare diseases. Collectively, these are estimated to affect 6-8 per cent of the population making rare diseases a public health priority. 
A study conducted in Western Australia (WA) found that in 2010, two per cent of the population had been hospitalised with a rare disease [5]. However, this group accounted for 10.5 per cent of hospitalisation costs in the same year [5], highlighting that people living with rare diseases can be intensive users of tertiary health services. In addition to an intense use within tertiary health services, people living with a rare disease often see multiple clinicians across the spectrum of primary, secondary and tertiary healthcare in the private and public sectors, as well as accessing disability and social services [6]. This means that there can be a range of health and other professionals involved in delivering care to people living with a rare disease. Responses to a survey of adults in Australia living with a rare disease [7] highlighted the multi-systemic nature of rare diseases with over one third (38 per cent) reporting that they saw three or more medical specialists in the last 12 months. In contrast, among adults in Australia living with any long-term health condition, just under one third (27 per cent) of condition saw three or more health professionals of any kind as opposed to specialist alone [8]. The need for people living with a rare disease to see high numbers of medical specialists not only reflects the multi-systemic nature of many rare diseases but the need for highly specialised knowledge; for people living with a rare disease in Australia, the main source of information about their condition was from a specialist (60.7\%) as opposed to a general practitioner (11.5\%) [7]. Despite their need to see numerous health professionals, less than one in ten respondents had a dedicated care coordinator [7].

In terms of their broader non-medical care, few respondents to the same survey agreed they received sufficient social (34.1\%), financial (14.5\%) and psychological (20.6\%) support [7]. Additionally, results from a European survey of people living with a rare disease found that most respondents considered that professionals from social services are poorly prepared to support them and that there is a clear lack of communication between service providers [9]. A recent Australian study has highlighted that seven out of 10 people living with a rare disease have unmet disability care needs [10].

Experiences of this kind have prompted calls for greater care coordination for people living with a rare disease $[11,12,13]$. An integrated approach to health care through care coordination-both within health systems and across agencies, is increasingly being recognised as critical to reducing fragmented care, improving the quality of care and patient experiences, lowering healthcare costs and improving sustainability, in health care more generally [14] as well as for rare diseases [15]. Importantly programs dedicated to improving the care coordination for people living with rare diseases such as the European Union-funded INNOVCare project are emerging [16]

While there have been several studies that explored care coordination experiences of individual rare diseases such as Huntington's Disease [17] and Sickle Cell Disease [18], few studies explore the experiences of those with a range of rare diseases. A recent and notable exception is a Canadian study exploring the care needs of children living with rare disease which has shed important insights into the care coordination experiences of this cohort [19]. However, studies that include the perspectives of adults living with a rare disease and which cover the Australian context, including those living in a regional location, are yet to be conducted. As a result, a key objective in the implementation of the WA Framework was to gather and publish evidence of the experiences of care coordination among people living locally 
with a range of rare diseases. In building an understanding on the care coordination experiences of people living with a rare disease, the results of this study make an important contribution to the rare disease literature.

\section{Methods}

Focus groups were selected as an approach to enable the researchers to develop an in-depth understanding of how people living with a rare disease experience care coordination and gain insights into the similarities and differences of these experiences through group interaction. A recruitment flyer was developed in conjunction with the WA based rare disease organisation, Genetic and Rare Diseases Network. Both the Genetic and Rare Diseases Network and the national rare disease organisation, Rare Voice Australia emailed the flyer to the patient support organisations and individuals registered with them, with Rare Voices Australia distributing to their Western Australia based members only. Both organisations also promoted the focus groups on social media. Opportunity to participate was also promoted in a Western Australian Department of Health news bulletin.

The flyer aimed to recruit participant for focus groups in the following categories:

- Focus Group 1: Parents of children living with a rare disease from metropolitan Western Australia

- Focus Group 2: Adults living with a rare disease (or their carers) from metropolitan Western Australia

- Focus Group 3: Parents of children living with a rare disease from regional Western Australia

- Focus Group 4: Adults living with a rare disease (or their carers) from regional Western Australia

Eligible participants were required to belong in one of the above categories, that is, be living with a rare disease, or caring for an adult or child living with a rare disease and reside in WA. Regional participants were those who lived outside of the Perth metropolitan area and were included to help capture differential experience; areas of regional Western Australia have amongst the world's lowest population density levels delivery of healthcare in regional Australia has well-recognised challenges [20]. Participants also had to be aged 18 years or over, have (or care for someone with) a confirmed diagnosis of a rare disease and have at least two health professionals involved in condition management. Registrants supplied the names of their conditions and these were checked against the Orphanet database of rare diseases (www.orpha.net). If the disease was listed in Orphanet the respondent was deemed to be living with a rare disease.

Six broad questions guided the focus group discussions, which covered:

(1) Exploration of the concept of "care coordination"

(2) A description from participants about how their care is currently coordinated

(3) Participants opinion on how well they think the health system enables care to be well coordinated for them 
(4) One change to the health system that would improve care coordination

(5) Perceived need for a professional to help with care coordination

(6) Participant opinion on whether this role could be fulfilled by a general practitioner.

In total, there were 19 eligible registrants and 16 final participants (see table 1 for more details). An unexpected result from the recruitment process was that the researchers received no registrants for Focus Group 3 for parents of children living with a rare disease from regional WA. Fourteen of the 16 participants provided their age, with age ranging from 30 through to 64 years and the average age being 47 years. Each focus group had at least one male participant and in total five males took part in the study; three of whom took part in focus group 2 for 'Adults (or their carers) living with a rare disease in metropolitan Perth', making this the only focus group with a balanced gender ratio. Thomas was the only carer of an adult living with a rare disease present and he provided care for his wife Denise who was also a participant.

Here - Table 1: Characteristics of the focus group participants

None of the participants within an individual focus group was managing the same rare disease. However, two conditions were shared across the entire sample and one participant was living with two diagnosed rare diseases; consequently, a total of 14 conditions were represented (see Table 1, column 7).

The focus groups were held in Perth, Western Australia. The metropolitan focus groups were undertaken face-to-face in a central location and the regional focus group took place using an online video and/or phone conferencing. Each focus group was scheduled to run for two hours inclusive of a 15 minute break. Focus groups 1 and 2 ran for longer with a total audio time of just over two hours for both discussions. The teleconference focus group was shorter at just under 1 hour and 45 minutes with no break. In total there was close to 6 hours of audio.

Each focus group discussion was recorded and transcribed verbatim by a third party. Thematic analysis was chosen as the method for analysing the focus group data and an essentialist approach within this methodology selected. This approach was selected to limit the amount that researchers look beyond or interpret what participants have said in the course of during focus group discussions and instead attempted to take what was said at face value. The analysis comprises six phases as outlined by Braun and Clarke [21] being (1) familiarising yourself with your data, (2) generating initial codes, (3) searching for themes, (4) reviewing themes, (5) defining and naming themes, (6) producing the report.

\section{Results}

The study revealed that people living with a rare disease (or their carers) (1) have complex care needs and a need to access multiple systems (2) have mixed experiences of care coordination (3) often go above and beyond to self-coordinate their care; and they (4) recognise opportunities for more systematic care coordination. 
Here - Figure 1: Themes and subthemes derived from qualitative analysis of interviews.

\section{Theme 1: Complex care needs and the need to access multiple systems}

Throughout the focus group discussions, participants described conditions that, while varying considerably in terms of their presentation, were all very serious in nature. This included multiple parents recounting that their child had been critically ill from birth and had spent time in neonatal intensive care. It also included descriptions of serious symptoms currently being experienced or that loomed into the future; these included renal failure, heart failure, blindness, hearing impairment or deafness, fatigue, anaphylaxis and anxiety. For some, there was a strong awareness that the condition was life limiting and, combined with the severity of symptoms and associated hardships, resulted in a necessity to live "from day to day".

Participants spoke of frequent contact with the health care system, such as with general practitioners, allied health professionals as well as hospital-based services such as specialist outpatient clinics, emergency departments and as inpatients. For many participants, conditions affected multiple body systems. As a result, there was often a "whole lot of different specialists" involved in their care and most respondents had many appointments to attend.

For many participants, the complexity associated with the serious and debilitating nature of their rare disease was compounded by low awareness of their condition by those directly involved in their care, especially in comparison to common conditions where there are "guidelines; the doctors know exactly what to do". Participants noted it was often the person living with a rare disease who knew more.

"Okay, I've got this." "What's that?" "Well you're in the medical world. You're supposed to know more stuff than us." Sometimes from the sounds of it, we all have our own information that we know more about than the doctors."

(Rachael, Adult from regional WA)

The seriousness of the rare disease, the frequency of contact with the health system and lack of knowledge of the condition within this system, create a complex medical situation for person living with a rare disease. Additionally, to meet their 'holistic' needs, participants described interactions with systems outside of health to access a range of social services. These include education services, mental health services and disability services, the latter of which can be a gateway to a suite of allied health services and other supports such as respite services.

\section{Theme 2: Mixed experiences of care coordination}

A minority of respondents described relatively good experiences of care coordination. This was largely due to having people within the health system who would assist with coordination, most often a general practitioner (GP) and other health professionals such as a specialist and clinical nurse. In addition, one respondent had a comprehensive care model and another a care plan. 
One participant living in regional WA described enjoying continuity of care over a period of 20 years within his local medical community and expressed a high level of satisfaction with his experiences of care coordination. Significantly, he stated that he would not consider moving to the metropolitan area for fear of losing this quality of care:

"I'm happy with the way basically everything coordinates. The hospital knows me, GP knows me, the specialist knows me, and I've only got to turn up and things happen. We never think about moving away purely because I know I can get the care coordination here that I get. I would shudder to try and get it in Perth."

(Peter, Adult from regional WA)

However, most respondents described at least some level of concern with the care coordination they received. This was expressed in various ways. Firstly, to meet the range of medical and non-medical care needs for the person living with a rare disease, participants described needing to navigate the complex health and other systems they interacted with and found considerable challenges in doing so. Postdiagnosis, they needed to 'fumble' along finding out for themselves what services they needed and could get access to. In relation to their medical care, participants frequently reported that they felt that they were not seen as 'a whole person' and that there was a lack of medical oversight of their condition. This lack of oversight often was in various forms including the absence of (i) a medical professional with an overview of their medical situation, (ii) an overarching medical plan; and (iii) a coordinated, multi-disciplinary team to deliver care. For some a lack of continuity of care with different doctors seeing the person living with a rare disease was a major issue as was a significant lack of forward planning amongst clinicians involved in their care, with the onus being placed on the person living with a rare disease.

"Even though the doctors should be managing it, I'm managing it and I'm really just using them as a sounding board to say, "Yep, you're on the right track, you keep going." (Sam, parent of child)

There were concerns about the health system being separated into medical specialities, where different specialists don't work or communicate well together. A major issue raised was the poor communication of critical medical information "between specialists and GP, and specialists and specialist" as well as others involved in health care management such as allied health professionals. Described by one participant as 'silo-isation in the medical field', the participant went on to describe a situation in which the responsibility to pass on critical medical information from one health professional to the other, falls to the person living with a rare disease. A related problem was the need to continually update health care professionals on medical history.

"So I'm then the one who has sole responsibility for passing on really quite complex information about quite complex health issues to the next specialist or the next health professional, and filling them all in. I just think it's just this enormous responsibility on individual patients and carers to be the sole repository of a whole lot of information from a whole lot of different specialists about a whole lot of different types of care when they're not all communicating with each other." (Melissa, adult) 
Another communication issue raised was the receipt of inconsistent information, which resulted in a lack of trust in health professionals.

"To then get completely conflicting information and yeah, so it's really then hard to - I found it really hard to trust the [health care] teams after that 'cause I was like, "Who's telling the right stuff and who's not?" (Fiona, parent of child)

Navigation challenges around non-medical supports were also described. These mainly related to access, finding appropriate supports as well as affordability. Participants spoke of "stumbling" across information to help support the non-medical care need for the person living with a rare disease or discovering supports, such as payments you are entitled to, "on the grapevine". Most participants described access to mental health support as important to them yet noted it was often stymied by factors as such as prohibitive costs and a lack of recognition of a need for support.

"In my experience has been that none of the people l'm seeing, none of the health professionals, not the specialists, not the GP, nor anyone else, none of them have said to me, "We think it might be an idea for you to go and see a psychologist."

(Melissa, Adult from metropolitan WA)

Accessing disability support services was a significant problem for some. As a range of allied health services as well as supports such as respite care are accessed through disability services, this had considerable implications for people living with a rare disease and family health and wellbeing. Parents reported a need to coordinate additional services, including education and childcare, and noted that the challenges here can be amplified by the need to adapt to different developmental stages in their child's life.

"Each section of their life is - you're thrown in a place with all this daunting stuff. There's so much stuff that needs to be coordinated at different times in their life."

(Grace, Parent of child)

One focus group discussed the health and other systems not being 'linked'. This referred to the fact that rare diseases might be recognised in one system such as health but not in other systems such as education and agencies providing government financial support, resulting in a requirement to complete forms for multiple agencies and to continually explain their situation.

\section{Theme 3: Going above and beyond to self-coordinate care}

Many participants saw the need to be proactive with respect to managing their care, and ultimately saw themselves, as well as those in their personal care networks, as their care coordinators.

"You are the coordinator of your own care. If you're well-informed, knowledgeable, you got well-informed, knowledgeable carers and family and support people, then we are in effect the care coordinators for our 
own care. It's not, that responsibility doesn't lie with our GP, or my hepatologist, or my whoever. It lies with me. So that's how care coordination actually occurs on the grass roots level." (Kathleen, Adult from metropolitan WA)

Self-coordination of care often involved coordinating and keeping track of multiple appointments and tests, which was time consuming and respondents became highly organised to manage this.

"For me, everything is on a square calendar on the fridge and every square is marked with what the heck is happening every day. So if there's an appointment, it's marked on a square on the calendar on the fridge door."

(Denise, Adult from metropolitan WA)

Participants also described dedicating a lot of time towards managing the non-medical care aspects of their care, for example gaining access to government payments.

"You sit in Medicare for two hours and then you sit in Centrelink for two hours." (Adam, Adult from metropolitan WA)

Participants adopted various strategies to assist in the coordination of their medical care. This included developing their own quasi-medical record by keeping copies of all their tests and results in a file and coordinating communication to ensure the medical team involved in their care was kept on the same page. One parent described a need to 'light the right fires' to try and encourage their child's medical team to work collaboratively, another described:

"So for me, my coordination was all coordinated by me, but it was about making sure I have all those connections and that I cc'd everyone into e-mail. I make sure I've got all their notes and they're always - I just annoy everybody really, I think. It's probably - It's the only way you can get it done." (Sarah, parent of child)

Many participants reported taking a leadership role in managing their condition. Significantly, one parent reported reviewing the latest research, writing a care plan for an aspect of their child's care which was then taken to and accepted by their child's doctors. For many others, this leadership involved making suggestions or prompting doctors about the next steps in their care.

"Then it's up to me to chase it up. Wait for another appointment and then say "Look, we haven't had a hearing test for a while", and then they'll go through the file and say "Oh yeah, it has been a while". (Anna, parent of child)

Participants also talked of needing to learn how the system works or to 'battle a way through the system'.

In order to help manage and coordinate their care, participants engaged in upskilling. For many participants, considerable time and resources had been spent developing an in-depth knowledge on their condition by reviewing medical literature (for those with access), searching information available on the 
internet and through patient support organisations as well as travelling long distances to attend conferences.

"I became Mrs Google - Everyone says, "Don't do Dr Google, don't do that," but thank goodness I did because I learned so much...Some of them kind of go, "That's crazy, don't read that." But by and large, you need to do a lot of your own research." (Kathleen, Adult from metropolitan WA)

The considerable efforts participants put into coordinating their medical and non-medical care were described as 'too time consuming', a pressure and responsibility that caused 'exhaustion', 'anger' and 'frustration' and having a physical and mental health impact. Those who had relatively positive experiences still found that coordinating aspects of their care to be extremely demanding. Many participants reported a burden of stress associated with coordinating their care and saw it as an add-on to a range of existing experiences of stress or concern including the frequently reported feeling of 'isolation' that comes from living with a condition many others do not understand. In addition, many participants described how the considerable time spent managing their condition affected the time that they could use "with the important things in life" such as time spent with children, or with siblings of a child living with a rare disease.

\section{Theme 4: Opportunities for more effective care coordination}

All focus group discussions raised the need for a person within the system who could be a central focal point, some-one with a big picture overview of the care requirements of the person living with a rare disease, invariably described as a 'case manager' or 'project manager'.

"Like someone other than me who has the overview of what I need, where I'm up to, and all the different treatments with all the different allied health professionals as well. Not just specialists but allied health, across the allied health." (Melissa, Adult from metropolitan WA)

The role of this person was seen to include: coordination of appointments and make sure these were well scheduled; helping to navigate the health system and other systems such as education and disability, providing information on where to go, what to do, who to call for help; linking the person living with a rare disease to needed services; collating information from specialists and other health professionals; and advocating for them in health and other systems.

"So I think just organising appointments that there's a lot that could be done for people who are having regular visits to the hospitals that their appointments are put within certain time spans, so that they can all be dealt with in a week or two. That would be wonderful, if you could just come down [from home hundreds of kilometres from Perth] and see all your specialists, boom, boom, boom."

(Joyce, Adult from regional WA)

Regarding the qualities of the person undertaking this coordination role, to advocate effectively for person living with a rare disease in complex systems it was felt by participants that they would need to be 
'bull terriers' and to have a passion for their role. In terms of their relationship with the patient and family, qualities seen as necessary included 'empathy' as well as a capacity to treat the patient's complexities as part of their job and not a burden. They were also expected to have a good knowledge of systems and services and of the health conditions that people were living with.

In terms of medical management, participants wanted greater oversight of their medical care to be in place. This could be in terms of at least one doctor being aware of the effect of the rare disease on the whole body or through having a multi-disciplinary medical team where everyone, including the family of the person living with a rare disease, is "on the same page".

"Meetings with the teams that are involved, allied health included. And then formulate a bit of a plan for what the family feels is important, the medical team, you know, kind of formulate that plan together rather than in isolation, within these little silos." (Fiona, Parent of child)

Taking a team approach, formulating a plan together, not working in silos and involving the person living with a rare disease and their families to ensure there are connections between all the relevant parties, including between medical and social services, were considered important.

"And not just coordination of care, of medical care, but coordination of life care, and psychological care, and home help, and who do you go to." (Melissa, Adult from metropolitan WA)

The qualities and the tasks undertaken appeared more important than the profession of the person to provide additional support. The role of the GP in supporting care coordination was mixed. For some participants the GP played an important role in managing their care and in some cases was helping to coordinate care. However, for the majority of participants, a GP was not seen as appropriate to undertake this role reasons including being 'out-weighted by specialists' and not featuring in the delivery of care to patients.

"You know, when you you've got complex needs, I think that you only see specialists." (Sophie, Adult from regional WA)

Participants also described the opportunity for better access across health and other systems to patient data and the advent of electronic medical records was welcomed as a potentially life-saving advancement.

\section{Discussion}

Our study has highlighted the complexity of care needs for many people living with a rare disease and the need for care coordination both within and across medical and non-medical domains. With respect to coordination of medical matters, a strong need was expressed by participants for a well-functioning team to manage care across various affected body systems; essentially support from a multidisciplinary medical team was sought. The important role of multidisciplinary teams in looking after a range of chronic conditions is well established [22] and is recognised as crucial to the diagnosis and management 
of rare disease in key planning documents including all three French national plans for rare diseases $[23,24,25]$ and the WA Framework. A couple of recent studies have explored more deeply the importance of multidisciplinary teams in the care provision of rare diseases requiring care from up to 10 different medical specialists being type I gaucher disease and tuberous sclerosis complex [26,27].

Both papers reported that it is essential to have (i) a care or treatment plan developed with the patient and/or family; and (ii) a physician taking a lead role for the delivering this plan. These requirements reflect the desire from focus group participants to have a care plan for their condition developed with their input, and to receive strong medical oversight for their condition. The Auvin et al study [27] reported that a care coordinator or case manager, such as a nurse of other professional, was considered the number one factor in enabling a multi-disciplinary team to function reflecting the difficulties perceived by clinicians in managing patient care.

In Europe, there is an array of infrastructure designed to improve the health and wellbeing of people living with a rare disease including centres of expertise. Using multidisciplinary teams as their cornerstone, these centres receive formal recognition for their capacity in the diagnosis and management of singular rare disease or groups of rare diseases and have built-on components including research (e.g. clinical trials) and education. While these centres have a focus on supporting medical care, infrastructure has also developed to address the coordination of non-medical care, such as education, disability, mental health and financial support, seen as vital by many focus group participants. In Europe these can be through initiatives known as 'resources centres' which provide supports that complement centres of expertise. These supports are wide-ranging and the result is a 'one-stop shop style' service which includes information and guidance services, training courses, provision of information concerning social benefits, daily support therapies, therapeutic recreation activities and in some cases medical and psychological consultations. They are intended to empower patients, families, carers and professionals and [28, 29]. While there are numerous formally recognised centres of expertise in Europe and to a lesser extent resource centres, they are less prevalent elsewhere in the world, including Australia.

While formalised networks of rare disease initiatives do not yet exist in Australia, individual programs supporting the same patient cohort and using the critical role of coordinators do. Exemplars include Victoria's Complex Care Hub [30] and WA's Undiagnosed Diseases Program [31]. A recent report from the McKell Institute in Australia has recommended a model of 'rare disease centres of excellence' that would include multidisciplinary teams of both health and disability care professionals to help counter the divide that exists between the health and disability sectors and the frequent need for people living with a rare disease to access both [10]. Similarly, the WA Framework has identified the need to explore the development of centres of expertise in the context of providing better care coordination for people living with a rare disease [2]. Findings from our study support suggest that changes are needed to improve current approaches taken to delivering care to people living with rare diseases. New approaches to be considered should include not only centres of expertise, but the complementary rare disease resource centres to ensure patients receive holistic, well-integrated medical and non-medical care. A key consideration in the development of these centres should be the roles of care coordinators or case 
managers and an allowance for diversity and flexibility of these roles acknowledging that some may be geared towards working behind the scenes to support multidisciplinary teams and others may be more outward facing, providing a named contact for people living with a rare disease that will help support their access to appropriate care. Better coordination can help to alleviate the time, stress and other burdens face by people living with a rare disease as they self-coordinate their care.

Moreover, improvements to the way in which care is provided to people living with a rare disease has the opportunity to lead to more resilient and more efficient health and welfare systems, while bringing about broad societal gains whereby people living with a rare disease are able to participate more fully in society [32].

\section{Conclusion}

The study's focus groups with 16 participants from both metropolitan and regional parts of Western Australia provides an important contribution to the limited literature on the care coordination experiences of people living with rare diseases. The paper addresses a research gap by capturing the views of both parents of children living with RD and adults living with rare diseases, and also explores some factors affecting people living in regional areas. The paper has highlighted that across all these groups, there are often significant care needs for people living with rare diseases across both medical and non-medical domains and that significant efforts are often undertaken to self-coordinate care. There are clear opportunities for more systematic approaches to care coordination including those using the key roles of multidisciplinary teams and care coordinators. Larger scale approaches that achieve multiple goals for $\mathrm{RD}$ patients including improved care coordination are worthy of serious consideration in an Australian context. These include centres of expertise for rare diseases and complementary resource centres.

\section{Limitations}

A significant limitation of the study was that parents of children living with a rare disease from regional Western Australia were not represented in the study. Further, demographic information collected from participants did not include data as to whether someone was an Aboriginal person, or from a culturally and linguistically diverse background. As a result, it was not possible to ascertain their representation in our findings. Capturing different perspectives in order to understand the diversity of experiences of care coordination is a priority and should be addressed by further research and engagement activities. Additional research that further explores the role of general practitioners in coordinating care is also recommended.

\section{Declarations}

\section{Ethics approval and consent to participate}


Ethics approval was obtained from the Western Australia Department of Health, Human Research Ethics Committee (approval number 2016/43). Participants provided consent to participate in signed consent forms.

\section{Consent for publication}

Not applicable.

\section{Competing interests}

The authors declare that they have no competing interests.

\section{Data availability}

Data resulting from our study is in the form of focus group transcripts. As this has the potential to identify our participants, for ethical reasons it cannot be made available to a third party.

\section{Funding}

The study was funded with in-kind support from the Office of Population Health Genomics.

\section{Authors' contributions}

$C M$ conceived of the study and $A B$ led the study design with contributions from $C M$. $A B$ and $C M$ facilitated the focus group discussions and analysed the transcripts. AB wrote a draft manuscript and $\mathrm{CM}$ provided critical revisions.

\section{Acknowledgements}

The authors would like to acknowledge the participants in this study and offer our sincere thanks to them for sharing their health care experiences. The authors would also like to thank the Genetic and Rare Disease Network, Rare Voices Australia and the WA Department of Health 'Health Networks' team for their support in the recruitment of participants.

\section{References}

1. European Commission. Rare diseases - what are they? 20135 July 2013 [cited 20137 July]; Available from: http://ec.europa.eu/health/rare_diseases/policy/index_en.htm. 
2. Department of Health Western Australia, 2015, WA Rare Diseases Strategic Framework 2015-2018, Department of Health, Western Australia: Perth, Western Australia.

https://ww2.health.wa.gov.au/ /media/Files/Corporate/Reports\%20and\%20publications/PDF/Rarediseases-strategic-framework.pdf

3. European Organisation for Rare Diseases (EURORDIS), 2005, Rare diseases: understanding this public health priority. EURORDIS.

4. Jaffe A, Zurynski Y, Beville L, Elliott E. Call for a national plan for rare diseases. Journal of Paediatrics and Child Health. 2009; 46:2-4.

5. Walker CE, Mahede T, Davis G, Miller, LJ, Girschik J, Brameld K, Sun W, Rath A, Segolene A, Zubrick SR, Baynam GS, Molster C, Dawkins HJS, Weeramanthri T. The collective impact of rare diseases in Western Australia, Genetics in Medicine, 2016; 19(5).

6. Department of Health Western Australia. Our children our future: A framework for child and youth health services in Western Australia 2008-2012. Department of Health Western Australia: Perth WA; 2008.

7. Molster C, Urwin D, Di Pietro L, Fookes M, Petrie D, van der Laan S, Dawkins H. Survey of healthcare experiences of Australian adults living with rare diseases. Orphanet Journal of Rare Diseases. 2016; $11: 30$.

8. Australian Bureau of Statistics. 0 - Patient Experiences in Australia: Summary of Findings, 2017-18. 2018. https://www.abs.gov.au/ accessed 9 August 2019.

9. European Organisation for Rare Diseases (EURORDIS). Juggling care and daily life: The balancing act of the rare disease community. A rare barometer survey. Paris, France; 2017.

10. The McKell Institute. Disability and Rare Disease: Towards Person Centred Care for Australians with Rare Diseases. New South Wales, Australia; 2019

11. Molster C, Youngs L, Hammond E, Dawkins H. Key outcomes from stakeholder workshops at a symposium to inform the development of an Australian national plan for rare diseases. Orphanet Journal of Rare Diseases. 2012; 7:50. Epub 10 August 2012.

12. Dharssi S, Durhane W, Harold M, Terry S. Review of 11 national policies for rare diseases in the context of key patient needs. Orphanet Journal of Rare Diseases. 2017; 12:63.

13. Rare Disease UK (RDUK), Rare Disease Care Coordination: Delivering Value, Improving Services, London UK; 2013

14. Sustainable Health Review. Sustainable Health Review: Final report to the Western Australian Government, Department of Health, Western Australia; 2019.

15. Hébert R. Applying integrated care systems to rare diseases, Expert Opinion on Orphan Drugs. 2016; 4:11, 1095-1097.

16. Castro R, Dan D, Tschank J, Sparring V, Vana I, Glinsner B. Bridging the gaps between health, social and local services, to improve care for people living with rare and complex conditions: key findings of the EU-funded INNOVCare project and its case management pilot. International Journal of Integrated Care. 2019; Vol.19(4).

Page 15/17 
17. Røthing M, Malterud K, Frich JC. Family caregivers' views on coordination of care in Huntington's disease: a qualitative study. Scandinavian Journal of Caring Sciences. 2015; 29:803-809.

18. Rattler TL, Walder AM, Feng H, Raphael JL. Care Coordination for Children With Sickle Cell Disease. American Journal of Preventive Medicine. 2016: 51(1), pp.S55-S61.

19. Currie G, Szabo J. "It is like a jungle gym, and everything is under construction": The parent's perspective of caring for a child with a rare disease. Child: Care, Health and Development. 2018; 45:96-103.

20. Australian Institute of Health and Welfare (AlHW). Australia's Health 2018. Australia's health services no. 16. AUS 221. Canberra: AlHW; 2018.

21. Braun V, Clarke V. Using thematic analysis in psychology. Qualitative Research in Psychology. 2008; 3: 77/101.

22. Taylor C, Munro AJ, Glynne-Jones R, Griffith C, Trevatt P, Richards M, Ramirez AJ. Multidisciplinary team working in cancer: what is the evidence? British Medical Journal. 2010; 340:c951.

23. Republique Francaise. French national plan for rare diseases 2005-2008: Ensuring equity in the access to diagnosis, treatment and provision of care. 2004.

24. Republique Francaise. National Plan for Rare Diseases (2011-2014): Quality of care for patients, research and Europe, a strengthened ambition. 2011.

25. Republique Francaise. French National Plan for Rare Diseases 2018-2022: Sharing innovation, a diagnosis and a treatment for all. 2018.

26. Torralba-Cabeza M, Olivera-González S, Sierra-Monzón J. The Importance of a Multidisciplinary Approach in the Management of a Patient with Type I Gaucher Disease. Diseases. 2018; 6, 69.

27. Auvin S, Bissler JJ, Cottin V, Fujimoto A, Hofbauer GFL, Jansen AC, Jóźwiak S, Kerecuk L, Kingswood JC, Moavero R, Torra R, Villanueva V. A step-wise approach for establishing a multidisciplinary team for the management of tuberous sclerosis complex: a Delphi consensus report. Orphanet Journal of Rare Diseases. 2019; 14/91.

28. European Organisation for Rare Diseases (EURORDIS). EURORDIS Policy Fact Sheet- Resource Centres, Paris, France. 2013.

29. Castro R, Senecat J, de Chalendar M, Vajda I, Dan D, Boncz B. EURORDIS Social Policy Advisory Group. Chapter 32 Bridging the Gap between Health and Social Care for Rare Diseases: Key Issues and Innovative Solutions in Rare Diseases Epidemiology: Update and Overview, Advances in Experimental Medicine and Biology 1031. Springer International Publishing AG . pp 605-627. 2017.

30. Stephens L, Mountford N, Bryan D. Simplifying complex care. In: Quality Initiatives - Entries in the $19^{\text {th }}$ Annual ACHS Quality Improvement Awards 2016. The Australian Council on Healthcare Standards. 2016.

31. Baynam G, Broley S, Bauskis A, Pachter N, McKenzie F, Townshend S, Slee J, Kiraly-Borri C, Vasudevan A, Hawkins A, Schofield L, Helmholz P, Palmer R, Kung S, Walker CE, Molster C, Lewis B, Mina K, Beilby J, Pathak G, Poulton C, Groza T, Zankl A, Roscioli T, Dinger ME, Mattick JS, Gahl W, 
Groft S, Tifft C, Taruscio D, Lasko P, Kosaki K, Wilhelm H, Melegh B, Carapetis J, Jana S, Chaney G, Johns A, Wynn Owen P, Daly F, Weeramanthri T, Dawkins H, Goldblatt J. Initiating an undiagnosed diseases program in the Western Australian public health system, Orphanet Journal of Rare Diseases. 2017; 12:83.

32. European Organisation for Rare Diseases (EURORDIS). Achieving Holistic Person-Centred Care to Leave No One Behind: A contribution to improve the everyday lives of people living with a rare disease and their families, Paris, France. 2019.

\section{Table}

Due to technical limitations, Table 1 is only available for download from the Supplementary Files section.

\section{Figures}

The experiences, understandings and insights into care coordination among people living with $\mathrm{RD}$ in WA (and/or their carers)
1. Complex care needs and the need to access multiple systems

2. Mixed experiences of care coordination

3. Going above and beyond to self-coordinate care

4 Opportunities for more systematic care coordination 1a.) participants have complex medical needs 1b.) participants have a range of non-medical care needs

1c.) difficulties with navigating systems to meet care requirements

2a.) some respondents described positive experiences

2b.) challenges with coordination of medical and non-medical care

3a.) participants assume a high level of responsibility in managing their care $3 b$.) consequences to these high responsibilities

4a.) the types of support that can lead to better care coordination

4b.) who can assist with care coordination

\section{Figure 1}

Themes and subthemes derived from qualitative analysis of interviews.

\section{Supplementary Files}

This is a list of supplementary files associated with this preprint. Click to download.

- Table1Participants.docx 